\title{
Auction-based Task Allocation for Teams of Self-Reconfigurable Robots
}

\author{
Zack Butler \\ Dept. of Computer Science \\ Rochester Inst. of Technology \\ zjo@cs.rit.edu
}

\begin{abstract}
Self-reconfigurable robots are versatile machines composed of many small, computationally independent modules. If a large number of modules is available, they can divide into multiple smaller robots instead of remaining as one large group. In such a case, they must decide based on their mission whether and when such a division is appropriate, and if so, how to divide the mission objectives amongst the smaller robots. Likewise, after division, a later task may be more effectively handled by merging two groups of modules to obtain a single robot with greater capability. For traditional teams of mobile robots, auction-based methods have been used for task allocation with good success. Here we develop an auction approach with new types of bids that incorporate splitting and merging of these robots. This allows the overall system to exploit its ability to vary the number of robots and their capabilities alongside allocation of the overall set of tasks. We apply this protocol to an exploration scenario, show its correctness and describe the results of simulation in several different environments.
\end{abstract}

\section{INTRODUCTION}

Self-reconfigurable (SR) robots are made up of many independent modules that can change their relative connections and/or topology to form robots of different shape and size. This ability can allow them to accomplish a variety of tasks that might not be achievable with a single fixedarchitecture mobile robot, such as climbing high obstacles and crossing gaps while also being able to navigate narrow passages. To date, a number of systems have been developed with various kinematics and capabilities (e.g. [7], [11], [12]; more details below). Within the context of these systems, we are interested in the potential that arises from being able to control the number of robots in a team. That is, a group of modules currently forming a single robot can potentially divide into two smaller robots, accomplish some tasks in parallel, then merge back together to accomplish a more challenging task. We refer to this general concept as a reconfigurable team to indicate that not only can the members of the team reconfigure, but the team itself can change its configuration to include varying numbers of robots with varying capabilities.

In our previous work, we developed reactive [1] and behavior-based [6] control methods for reconfigurable teams. The reactive method is a computationally simple method that is successful in simple environments (where tasks do not often require long-term planning and merging of robots) but can fail in more challenging scenarios. In contrast, the behavior-based technique was successful in a number of environments, but relies on specifying motivation levels for a number of behaviors which leads to complex interactions among them. This makes it hard to guarantee the overall team performance or add new behaviors to the system.

In this work, we have taken inspiration from the significant work in auction-based task allocation techniques that have been developed for robot teams (e.g. [3], [5], [8]). In these methods, new tasks are given to the robot team (or generated by the robots themselves) and allocated to specific robots through a bidding process. In general, each robot bids based on its cost to accomplish the task and the reward defined by the task. Auction-based techniques have been shown to have good results in a variety of scenarios, and can be robust to loss of communication and loss of robots. Our contribution is to extend the auction concept to take advantage of the reconfigurability of the team. Specifically, robots will be able to make bids that take into account splitting up or merging together - for example, a robot that splits into two can potentially take on two tasks in parallel, and this would be reflected in its cost calculations. This paper presents the new types of bids that we have developed for this scenario, along with an analysis that shows that the enhanced protocol will lead to successful completion of all tasks. The application presented here is one of exploration, in which the tasks are locations to visit at the edge of explored space, known as frontiers. However, the overall framework and concept of bids that use splitting and merging can be extended to other types of tasks.

Our work also has parallels with work in auctions for coalition formation, since a bid that involves merging two robots is similar to robots forming a coalition. However, in that work, the focus there is usually on the qualitative requirements of the task, where the task can be decomposed for assignment [14] or coalitions are made based on collecting information of individual robots' capabilities [13] In our case the merged robot will have capabilities that are quantitatively more than the union of the capabilities of the constituent parts, and will act as a single robot after the merger, as opposed to two cooperating entities.

Finally, it is important to note that this research does not consider how the individual self-reconfiguring modules coordinate to produce motion, division, or merging. These techniques are necessarily dependent on the underlying hardware, and a variety of systems have been developed that have some or all of the relevant capabilities. SR robots have been developed both as lattice-based systems, in which the modules form a dense lattice that can reconfigure into 
different shapes; chain-based systems, in which modules link together to form chain, loop and tree-like structures; and hybrid systems that can work in both modes. Motion over uneven ground has been demonstrated for the hybrid MTRAN system [7] and a generic simulated model of latticebased modules [4], among others. Division and merging of groups has been shown in simulation for lattice-based systems [2] and in practice on the chain-based CONRO system [10] and CkBot system [12]. From another point of view, the Swarmbots project [9] has developed small wheeled mobile robots that are capable of independent locomotion but can also physically join together to create more capable larger groups (for example, to reach across a gap in the floor larger than one individual robot). It is our intent that the technique developed here is applicable to any such system that is capable of locomotion, division and merging. The simulation described in this work looks at complete robots (groups of modules) and is designed to allow easy replacement of motion and sensing models so that systems with different capabilities can be both planned for (using the models to calculate bids) and executed (using the models to generate simulated motion and sensing).

\section{Auctions for Reconfigurable Teams}

In standard auction-based task allocation, an auctioneer (either one of the robots in the system or an external agent) announces a task to the group, and each robot in the team then has the opportunity to bid on the task. Each robot considers the task and calculates an amount it is willing to bid as the amount it expects to gain for the team (for example, the amount of area it expects to survey given its sensors) minus its cost to reach and execute the task, such as the expected time or energy required (appropriately scaled, since it will generally not be in the same units as the benefits). The auctioneer accepts bids for a specified amount of time, and at the end of this time, will choose the highest bid and inform all bidders of the winner of the auction. The timeout is required in case of loss of communication or if a robot chooses not to bid in a given auction.

\section{A. Bid Types}

In order to use auctions with reconfigurable teams, we will need new types of bids that incorporate the possibility of splitting or merging. These will be in addition to the regular bids in which one robot computes its profit for a particular task. From a basic standpoint, a single robot may offer to split up and take a new job in parallel with its currently assigned $\operatorname{task}(\mathrm{s})$, or it may try to find a colleague with which to make a common bid based on the two robots merging together.

For a regular bid in our system, we use traditional notions of cost and benefit to come up with a bid value. The cost can be computed in a variety of ways, as in previous implementations of auction protocols. In our implementation we have used the minimum additional time that the robot would take to insert the new task within its current agenda. If the robot has no tasks, this is just the time to accomplish the task. The benefit is quite task-dependent and can vary based on the overall mission goals; for exploration we tested both estimated sensed area and a constant benefit. The results in Sec. V show the latter, but the two cases gave very similar results since the robots have similar sensing capability.

For a split bid, the robot will decide whether it is more efficient to split itself to accomplish the task up for auction in parallel with its current $\operatorname{task}(\mathrm{s})$. A single robot will never make both a regular bid and a split bid in the same auction; it will determine for itself which is more profitable and only submit the better bid. To compute the cost of a split bid, the robot divides the new and current tasks into two groups and compares the worse time-to-completion of these two groups (given the smaller robots) to the current timeto-completion of its task list. If the tasks can be performed in parallel by the smaller robots after the split, this value could be zero. However, we do impose some limitations on splitting. First of all, depending on the system, the splitting process itself will likely be non-trivial, so an additional cost is added representing the time taken to perform the split. We can also heuristically increase this split cost further to discourage excessive splitting — if too many small robots are created, it may make future execution less efficient due to their reduced capability. We explore the effects of this heuristic choice in Sec. V. We can also set a minimum size (number of modules) for a robot. Currently, we only allow a robot to make a split bid when it has exactly one current task, so that if it won the auction it would have two tasks to give to the two robots that it splits into. While this is restrictive, our reasoning is that if the robot already has multiple tasks and has not yet split, it would not be efficient to accomplish those tasks after a split. This also avoids having to compute the best possible split of a potentially large number of tasks.

For a merge bid, two robots must collectively generate the bid before submitting it to the auctioneer. For the cost computation, the robots have to consider how long it will take for the single merged robot to achieve all the tasks that both currently hold, including the one up for bid. Of course, if neither robot can achieve the auctioned task alone, then a merge will be their best option for this task, even though it may not be efficient to perform the merge right away. In our implementation, rather than consider all possible merges, a robot will offer itself for a merge by sending a message, and consider such offers, only if it has no tasks currently assigned. This may reduce efficiency but will not affect completeness, since if no single robot can accomplish the task up for auction, it will be auctioned again later on, and once the current robots finish their tasks, they will then propose a merge. Since a robot may get multiple offers to merge, it waits for a specified period of time before making its bid based on the best offer it received (if any). The cost of achieving the task via a merge is then the time taken for the robots to reach a common location, perform the merge, and then go to the task location. To avoid exhaustive computation, the robots consider meeting up only at the offering robot's current location (note that each robot will make an offer to the other). As with splitting, the cost of the merge itself can be set based on the capability of the underlying system. 


\begin{tabular}{|l|l|l|}
\hline Message type & Payload & When used \\
\hline \hline AUCTION & Task ID, location $(\mathrm{x}, \mathrm{y})$ & $\begin{array}{l}\text { By auctioneer } \\
\text { to initiate auction }\end{array}$ \\
\hline BID & Task ID, bid amount & In response to AUCTION \\
\hline OFFER & Task ID, offerer location & $\begin{array}{l}\text { By potential bidders } \\
\text { to find merge partners }\end{array}$ \\
\hline $\begin{array}{l}\text { AUCTION- } \\
\text { COMPLETE }\end{array}$ & Task ID, Winner ID & $\begin{array}{l}\text { By auctioneer } \\
\text { at end of auction }\end{array}$ \\
\hline \hline STATUS & Location, map data & Periodically by all robots \\
\hline
\end{tabular}

TABLE I

Message tyPes USED IN THE SYSTEM. NOTE THAT ALL MESSAgeS ALSO IMPLICITLY INCLUDE THE IDENTITY OF THE SENDER.

\section{B. Additional restrictions}

Since the auction process will be distributed (that is, any robot can act as an auctioneer), and a single sensor sweep can generate multiple frontiers, it is likely that multiple auctions will overlap in time. In traditional auction-based task allocation, there are different ways that this is handled. One is through combinatorial auctions (e.g. [8]), in which each robot can bid for multiple tasks as a single lot, but this is quite complex even for traditional robots. Another option is that each auction is bid on independently, acknowledging that this may result in an overall inefficiency if a robot wins several auctions. For SR systems, the latter approach is also problematic, as (for example) making a succession of independently calculated split-based bids can be very optimistic relative to the true cost. Thus, we allow each robot to have only one outstanding split-based or merge-based bid at any time, and not to make bids in new auctions unless they do not conflict with bids in open auctions. In addition, each robot will only act as auctioneer for one auction at a time to minimize the constraints that this imposes.

\section{EXPLORATION USING AUCTIONS}

To perform exploration, a group of SR robots will travel through an initially unknown environment, discovering new locations to explore and using the auction protocol to allocate these locations while splitting and merging to increase efficiency. When a robot detects nearby unexplored area, it will create and auction one or more tasks based on the frontier between explored and unexplored area. To minimize overlap between tasks, the creation of one frontier will suppress generation of nearby tasks until it is reached.

We have implemented the cooperation between robots, including auctions, through a message-passing protocol. The various message types used are described in Table I. The auctions themselves use four types of messages, and an additional STATUS message is sent periodically so that all robots will maintain reasonably up-to-date knowledge of the team's progress and current map.

As the robots move about the environment, they execute the control loop given in Algorithm 1. Each robot maintains an ordered list of tasks (locations to visit) while also handling its own auctions and bidding on auctions held by its colleagues. If its initially planned route turns out to be impassible, it will try alternative routes, and if this fails, it will queue the task for eventual reauction.

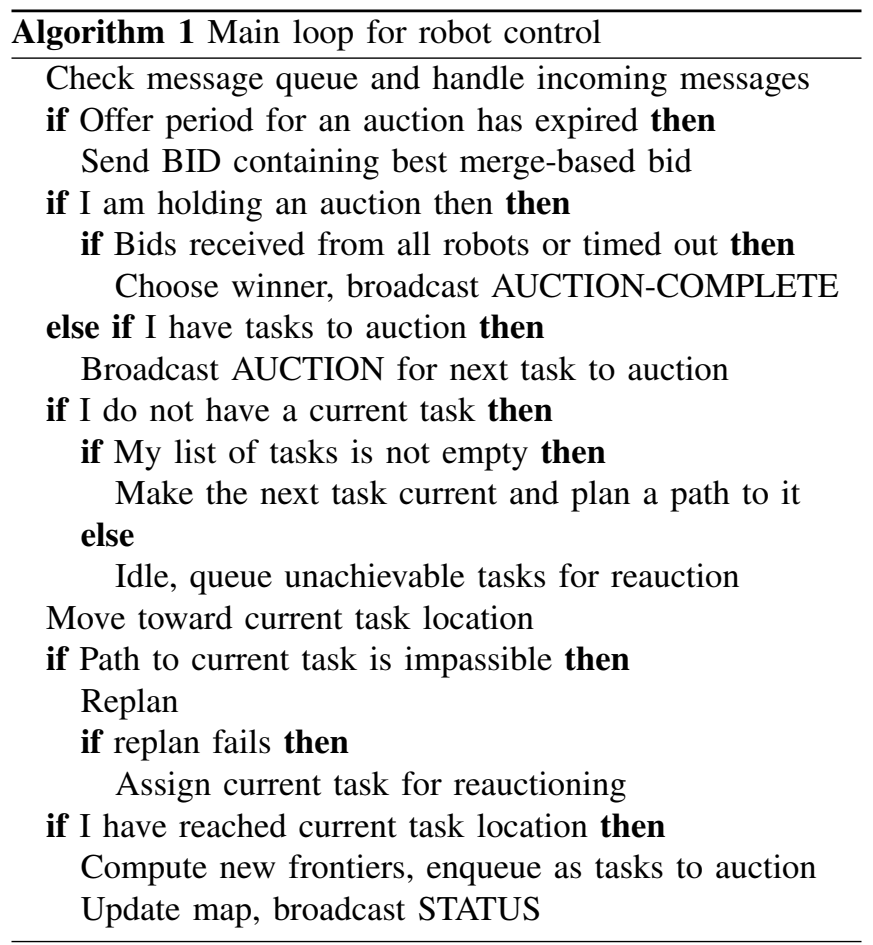

The bidding process itself is dealt with in the relevant message handlers. These are shown in Algorithm 2. The AUCTION handler computes the costs for a regular bid and (if available) a split bid, and responds with a BID message containing its best option. Since the benefit is considered the same for all robots, we simply use a constant. To compute the cost of a split bid, the robot must hypothesize the ability of the robots that it will split into for the correct path planning to take place. Finally, if the robot cannot reach the task at all, it will instead send an OFFER message.

The OFFER handler runs on all robots to consider a joint bid. Again, the robot determines the capabilities of the hypothetical merged robot and computes the correct path and bid cost. Even if the merged group cannot reach the task location, a merge bid is still developed, as it may be necessary for two robots to merge first before merging with a third to be able to reach the desired location. Note that the OFFER handler does not send a BID message - instead it locally stores the best merge bid including the identity of the robot it would merge with. Only when the offer timeout has expired will it submit a merge bid based on the best offer received. Therefore, it is necessary that the offer timeout is significantly shorter than the auction timeout.

Finally, the AUCTION-COMPLETE handler will run in response to the end of an auction. Robots that did not win will simply take note of the end of the auction - since we impose some restrictions on what bids can be outstanding at any given time, it is useful to know when an auction is over, though not strictly necessary since the auctions are guaranteed to time out. The winner(s) of an auction will take on the task. In the case of a regular bid, the task will be added to the robot's list of tasks. Rather than reordering 


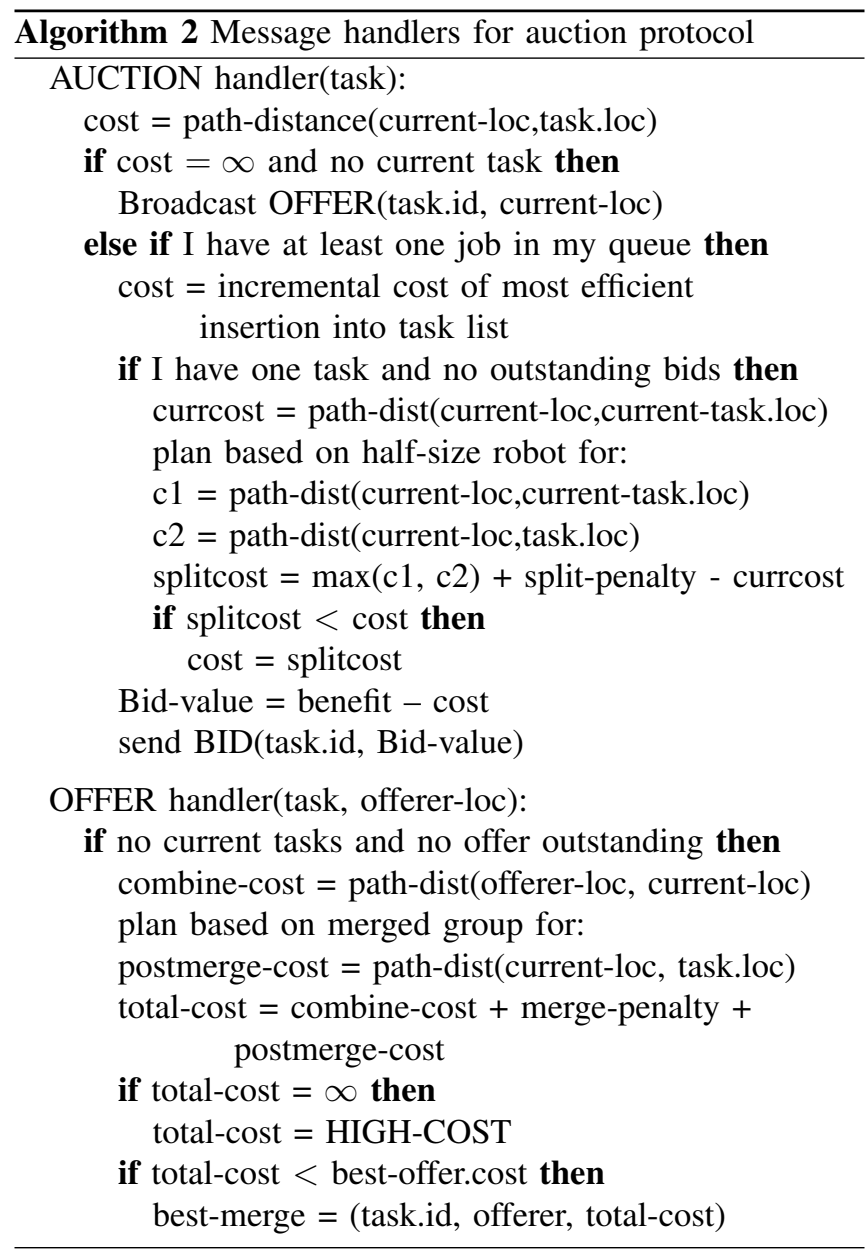

the entire list of tasks, which is equivalent to the Traveling Salesman Problem, the robot simply inserts the new task at the best possible location in its agenda, as has been done in previous auction protocols [15]. With a split bid, the robot will immediately split, giving its current task to one of the new smaller robots and the new task to the other. For a merge bid, the robot making the bid will put the task in its queue but wait at its current location while its helper will move to that location to complete the merge. Once the merge is complete, the task will be transferred to the new larger robot.

\section{Protocol Analysis}

The goal of our analysis is to show that the auction protocol will lead to complete exploration by a reconfigurable team. Since each frontier reached will either lead to further frontiers or uncover the boundary of the environment, if every frontier auctioned off will eventually be reached, then the environment will be fully explored. The critical point is to show this in the case of splitting and merging, where the capabilities of robots can change dynamically, potentially even during the course of an auction. We will first show that this is true when one auction takes place at a time, and then extend this result to the more realistic case of overlapping auctions. This analysis assumes that a robot will not completely fail in attempting to reach its goals. This is reasonable for an SR robot - if instead some individual modules fail, this would result in a smaller, perhaps lesscapable group, but that group would still be around to auction off any goals that it now cannot reach.

\section{A. Single auction analysis}

For a single auction, we consider the type of bid made by the winner of the auction and what other tasks it had before the auction. We present case-by-case analysis based on the number of tasks already assigned to the winner.

If the winner has no other tasks before the auction, it cannot make a split-based bid, so must be either a regular (solo) bid or a merge-based bid. In the regular case, with no other tasks to worry about, it will go directly there. The only exception is if no robot can reach the task, in which case the auctioneer will hold it for later reauctioning, as described below. In the case of a merge-based bid, one of the two robots on the winning coalition will wait while the other moves toward it. These robots would not make such a bid without being able to reach each other, and with no other tasks on their agenda, their situation will not change during the auction period. Thus, they will move toward the merge and then toward the new task location.

If a robot has one task on its agenda when the auction occurs, it will not make a merge-based bid, but can make a split-based bid. If so, upon winning the auction, it will split and send one of its successor robots to the new task (it would not have made a split bid if this were not feasible). There are two exceptions: if its original task was achieved during the auction period, it will take the new task without splitting; second, if it has moved to a point where the smaller robots will no longer be able to reach the new tasks, it simply takes the new task without splitting. For a regular bid made with one task already on the agenda, this second task will be added either before or after it, but in either case a series of path segments will exist to reach both goals.

Finally, with more than one task on the agenda, only regular bids can be made, and again the ability to reach the task will not change during the auction period, so the task will be reached or put up for later reauction.

\section{B. Simultaneous auctions}

When two or more auctions are held at the same time, it becomes possible for a robot's status to change significantly between the time it makes its bids and the time that it wins the auctions. For example, consider a robot that makes a regular bid and a split bid in two different overlapping auctions. If it wins the split bid first, it immediately splits into two, but if it then wins the regular bid, this winning bid may be unable to be executed since it was based on the larger group size. Again we will break down the cases by the number of bids held before the auctions, but now we look at combinations of winning bids instead of single bids.

With no current tasks, a robot may make multiple regular bids and/or one merge-based bid. With only regular bids, though the bids may be optimistic, winning all of them will not interfere with achieving all the tasks. If a merge-based 

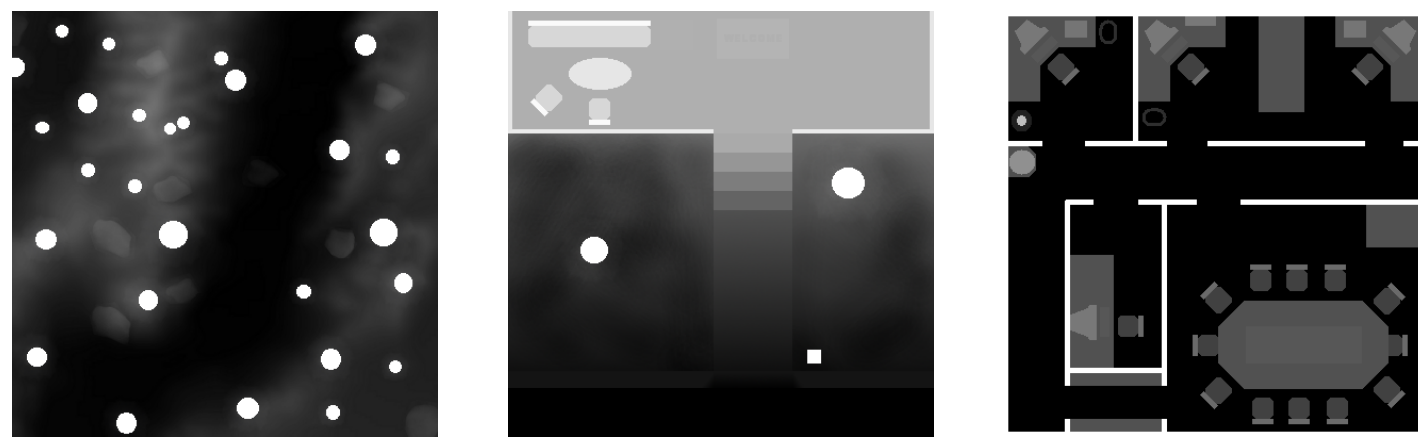

Fig. 1. Height maps of three simulated environments, in which black is the lowest altitude and white is high enough to be non-traversable. (Left) A forest, in which circles represent tree trunks. (Center) A porch with high railings with a flat area in front. (Right) An office with high walls and furniture.

bid is made, however, it may be awarded alongside a regular bid. If the regular bid is awarded first, the winner will start moving, and potentially make the merge impossible. Thus, the merge bid is checked before being added to the list of tasks and the task reauctioned if necessary. If the merge is awarded first, it will take priority, and the other task will simply be added to the tasks of the merged robot. Since the larger merged robot will be at least as capable as the original bidder, this task will still be achievable. By not allowing a robot to make a second merge-based bid (or offer its services to another robot) if it has a merge bid outstanding, we avoid a deadlock situation where two robots are each waiting for the other to arrive and merge.

With one task in its queue, a robot can make regular and split-based bids. Winning a split bid and then a regular bid can be problematic since the regular bid was based on the capability of the larger robot. Likewise, winning two split bids could give a robot tasks that can not be achieved. Thus, it is not allowed to make a split bid with any other bid outstanding, and so all assigned tasks will be achievable.

Finally, with more than two tasks to do, all that a robot can do is make a number of regular bids, and if it wins, will add all of those tasks to its queue. Even if the bids are too optimistic because the costs were calculated independently, all tasks will be achievable since the bids were based on the capability that still exists.

\section{Reauctioning}

Because of the nature of self-reconfiguration, it is possible that a valid task is given that cannot be achieved with the current collection of robots. For example, during exploration a group of modules may be able to see a step that neither it nor any other current robot can climb up but that they could climb if merged. In this case, when the task is auctioned, either a merger will be proposed, or there will be no successful bid. In the latter case, it will remain with the auctioneer to auction again at a later time. This will not prevent the task from eventually being completed - once all achievable tasks have been completed, there will be two or more robots with nothing to do, and these can then propose a merged bid. This will eventually lead to all frontiers being reached. For efficiency, we can also allow robots to periodically reauction tasks that are in their queue, as is done in some existing auction protocols. We would then have to make sure that each task will eventually be reached by some robot even if it can be auctioned repeatedly, which depends on the specifics of the bid calculations. These opportunistic reauctions were thus not included in the experiments reported below, though we have included them in other tests without any failures.

\section{IMPLEMENTATION AND RESULTS}

We have implemented the auction protocol within our previously developed simulator for SR robots. As mentioned earlier, it does not simulate individual modules, instead concentrating on the behavior of groups of modules to make it applicable to a variety of systems. To reinforce this concept, the sensing and motion models can be easily swapped out with new ones specific to a given system. The control can also be swapped out to easily compare different algorithms. Here we used a generic motion and sensing model. For motion, a robot can climb any obstacle below a given relative height, where this maximum height is linear in the number of modules present. For sensing, each robot can sense the environment along lines of sight in a radius proportional to the cube root of the number of modules (modeling an approximate hemisphere of modules).

The protocol was tested in four environments, each of size $400 \times 400$ units (the distance unit is defined as the diameter of one module). The simulation timesteps are then set such that a robot can move 1 unit of distance for each time step of the simulation. Three environments are shown in Fig. 1, with the fourth a flat plane. In all experiments, the modules start as a single robot in the center of the environment, as we expect most applications will use a single point of deployment. The initial robot consisted of 1400 modules, though again this is really only meaningful relative to the motion capability for example, in the office environment, the tables and desks require a robot of at least 500 modules to surmount.

For each experiment, we recorded the time taken, the total distance traveled by all robots and the total distance traveled weighted by the size of each robot. These latter two metrics each measure power consumption, and either may be more relevant depending on the type of hardware. We investigated different values of the split cost as mentioned earlier to see how this would effect the overall performance 


\begin{tabular}{|c||c|c|}
\hline Method & Flat environment & Forest environment \\
\hline Auction, sc $=0$ & $1634 / 5654 / 1050$ & $2667 / 9013 / 1930$ \\
\hline Auction, sc $=50$ & $1023 / 5281 / 969$ & $2354 / 8046 / 1700$ \\
\hline Auction, sc $=200$ & $2247 / 5059 / 2383$ & $3979 / 7714 / 4631$ \\
\hline Auction, sc $=500$ & $3815 / 4217 / 5904$ & $6486 / 7393 / 10351$ \\
\hline Behavior-based & $1249 / 11217 / 19353$ & $1493 / 13219 / 2486$ \\
\hline
\end{tabular}

\begin{tabular}{|c||c|c|}
\hline Method & Porch environment & Office environment \\
\hline Auction, sc $=0$ & $3936 / 11026 / 3973$ & $7497 / 14400 / 9982$ \\
\hline Auction, sc $=50$ & $4874 / 12622 / 5585$ & $7860 / 14900 / 10127$ \\
\hline Auction, sc $=200$ & $6431 / 9239 / 7967$ & $6110 / 6980 / 8670$ \\
\hline Auction, sc $=500$ & $6296 / 7126 / 9914$ & $5607 / 6319 / 8562$ \\
\hline Behavior-based & $3011 / 13883 / 4683$ & $5571 / 13832 / 8596$ \\
\hline
\end{tabular}

TABLE II

PERFORMANCE OF AUCTION-BASED EXPLORATION WITH VARYING

SPLIT COST (SC), COMPARED TO A WELL-TUNED BEHAVIOR-BASED

APPROACH. EACH CELL LISTS TIME (IN SIMULATOR TIMESTEPS), TOTAL DISTANCE TRAVELED BY ALL ROBOTS (IN UNITS OF MODULE SIZE), AND TOTAL DISTANCE WEIGHTED BY ROBOT SIZE.

of exploration. Table II shows the results for different environments and different values of the split costs, along with results of our behavior-based approach. The split cost is in the same units as the bids, which in this case is equivalent to simulator distance units. As mentioned, the split cost can be defined by the hardware capability, but we also heuristically increase it by varying amounts to take into account future challenges of having smaller, less capable individual robots. As expected, in simpler environments, increasing this beyond a nominal value slows the system down as it overly discourages parallelism. On the other hand, in the office environment, which has many tall obstacles that require large robots to climb on, this discouragement makes the overall exploration process more efficient. In general, compared to the behavior-based approach with carefully tuned parameters, the auction approach was much more power-efficient, and similar in terms of time efficiency with an appropriate split penalty. The largest split penalty was enough to discourage splitting in virtually all cases, so this can also be seen as approaching the performance of auctioning without using self-reconfiguration. In most of the environments, this was clearly the worst option, but in the challenging office environment it was actually most efficient to avoid splitting up, as it inevitably led to merging back together with the inherent additional time required.

\section{Discussion}

In this paper we have investigated a new approach to take advantage of the potential of self-reconfiguring robotic systems. Leveraging the existing auction-based approach has allowed us to develop an algorithm that efficiently and completely explores unknown environments. We have also shown that different environments may reap quite different amounts of efficiency gains from the ability to divide and merge robots. This is likely to be true regardless of how tasks are allocated, and so recognizing the character of the environment and responding appropriately is an important avenue of investigation.

Our other efforts to extend this work involve expanding the auction protocol to cover other types of tasks, such as placement of surveillance sensors during exploration, and to heterogeneous systems, in which various SR robots may contain different types of modules, such as different sensors. Traditional robot task allocation has shown a variety of ways to approach these problems by modifying the ways in which robots bid on tasks. Using these algorithms as inspiration, we should be able to incorporate these variations within our bidding framework while retaining correctness.

Finally, while we have focused here on the interaction between entire SR robots, we are also developing simulations that integrate these algorithms with those that handle the control of individual modules. By developing a complete system of hierarchical distributed control at both the module level and robot level we can help bring these systems to a point where they fully live up to their potential.

\section{Acknowledgments}

We gratefully acknowledge Jacob Hays for the original simulator that was used as the basis for the simulations presented in this paper.

\section{REFERENCES}

[1] Z. Butler and E. Fabricant. Reconfigurable teams: Cooperative goalseeking for self- reconfigurable robots. In Distributed Autonomous Robot Systems, 2008.

[2] Z. Butler, S. Murata, and D. Rus. Distributed replication algorithms for self-reconfiguring modular robots. In Distributed Autonomous Robotic Systems 5, pages 37-48, 2002.

[3] S. Jain C. Tovey, M. Lagoudakis and S. Koenig. The generation of bidding rules for auction-based robot coordination. In F. Schneider L. Parker and A. Schultz, editors, Multi-Robot Systems: From Swarms to Intelligent Automata, volume 3, pages 3-14. Springer, 2005.

[4] Robert Fitch and Zack Butler. Million module march: Scalable locomotion for large self-reconfiguring robots. The International Journal of Robotics Research, 27:331-343, 2008.

[5] Brian P. Gerkey and Maja J Mataric. Sold!: Auction methods for multi-robot coordination. IEEE Trans. on Robotics and Automation, 18(5):758-768, October 2002.

[6] J. Hays and Z. Butler. Hierarchical distributed task allocation for multi-robot exploration. In Distributed Autonomous Robot Systems, 2010.

[7] A. Kamimura, H. Kurokawa, E. Yoshida, S. Murata, K. Tomita, and S. Kokaji. Automatic locomotion design and experiments for a modular robotic system. IEEE/ASME Trans. on Mechatronics, 10(3):314-325, 2005.

[8] Liu Lin and Zhiqiang Zheng. Combinatorial bids based multi-robot task allocation method. In Proceedings of ICRA, pages 1145-1150, 18-22 April 2005.

[9] F. Mondada, L.M. Gambardella, D. Floreano, S. Nolfi, J.-L. Deneubourg, and M. Dorigo. The cooperation of swarm-bots. IEEE Robotics \& Automation Magazine, pages 21-28, June 2005.

[10] M. Rubenstein, K. Payne, P. Will, and Wei-Min Shen. Docking among independent and autonomous conro self-reconfigurable robots. In Proceedings of ICRA, pages 2877-2882, 2004.

[11] Wei-Min Shen, Maks Krivokon, Harris Chiu, Jacob Everist, Michael Rubenstein, and Jagadesh Venkatesh. Multimode locomotion for reconfigurable robots. Autonomous Robots, 20(2):165-177, 2006.

[12] Babak Shirmohammadi, Camillo J. Taylor, Mark Yim, Jimmy Sastra, and Mike Park. Using smart cameras to localize self-assembling modular robots. In Proceedings of the 1st ACM/IEEE International Conference on Distributed Smart Cameras, pages 76-80, 2007.

[13] F. Tang and L. Parker. Distributed multi-robot coalitions through ASyMTRe-D. In Proceedings of IROS, pages 2606-13, 2005.

[14] R. Zlot and A. Stentz. Market-based multirobot coordination for complex tasks. Int'l J. of Robotics Research, 25(1):73-101, 2006.

[15] Robert Zlot, Anthony Stentz, M. Bernardine Dias, and Scott Thayer. Multi-robot exploration controlled by a market economy. In Proceedings of ICRA, pages 3016-3023, 2002. 